\title{
Performance and Analysis of Automatic Detection of Ground- Glass Pattern in Lung Disease using High-Resolution Computed Tomography
}

\author{
M. Anto Bennet*, G. Sankar Babu**, S. Mekala***, S. Natarjan****, N. Srinivasan***** \\ $*, * *, * * *$ Department of Electronics and Communication Engineering, Veltech, India \\ $* * * * * * * * *$ Department of Electrical and Electronics Engineering, Veltech, India
}

\begin{tabular}{l} 
Article Info \\
\hline Article history: \\
Received Jun 18, 2015 \\
Revised Aug 18, 2015 \\
Accepted Aug 27, 2015 \\
\hline Keyword: \\
Chest Radiograph (CXR) \\
Computed Tomography (CT) \\
CXR findings \\
Discrete Wavelet Transform \\
(DWT) \\
Gabor filter, Threshold (T) \\
Multi-Detector (MDCT) \\
Post processing \\
Preliminary mask
\end{tabular}

\begin{abstract}
This study proposes an approach for automatic detection of Ground glass pattern, a lung disease, from Computed Tomography (CT) and High Resolution Computed Tomography (HRCT) scans of the lung. The algorithm is based on frequency spectrum analysis of image using Gabor filter bank. Gabor filter banks are used to support the frequency extraction process. These algorithms when applied to HRCT images will assist doctors to gain more information than from the CT images. The tasks are completed in three steps: Preliminary mask formation, Peripheral mask formation and finally post processing. By these, higher sensitivity and selectivity may be achieved with fast processing time. In the post processing, binary noise removal technique is used to remove noise from the detection mask.
\end{abstract}

Copyright $\odot 2015$ Institute of Advanced Engineering and Science. All rights reserved.

Corresponding Author:

M. Anto Bennet,

Department of Electronics and Communication Engineering,

Veltech,

Avadi-Chennai-600062, Tamilnadu, India.

Email: bennetmab@gmail.com

\section{INTRODUCTION}

High Resolution Computer Tomography (HRCT) is the radiological imaging technique currently available that most closely reflect changes in lung structure. It represents the radiological method of choice for the diagnostic work-up of patients with known or suspected diffuse interstitial lung disease. It has clearly been shown to be superior to chest radiography and standard thick section CT for detecting and clarifying the pattern and extent of parenchyma lung disease. HRCT is capable of producing anatomic information similar to that obtained from pathologic examination of lung sections. The technique of HRCT was developed with relatively slow CT scanners, which did not make use of Multi-Detector (MDCT) technology. The parameters of scan duration, z-axis resolution and coverage were interdependent. For a conventional chest CT scan, in order to cover the chest in a reasonable time period, it was necessary to use thick sections (e.g., $10 \mathrm{~mm}$ thick) in order to ensure continuous coverage. As performing contiguous thin sections would require unacceptably prolonged scan time, HRCT examination was therefore performed with widely spaced sections.

Localization by HRCT was defined as ground-glass opacities and/or alveolar consolidation; the latter abnormalities were considered to reflect the filling of the alveolar lumen with blood. In the absence of alveolar filling, isolated cavitations and/or a mass were considered to be localizing lesions. Interpretation of the HRCT findings must take into consideration possible redistribution of blood to dependent portions of the lung. For this reason, when ground glass opacities were present in both the upper and lower lobes, areas with 
more attenuation were considered the bleeding site and, in cases of homogeneous attenuation, the upper lobes were considered to be the bleeding site. The bleeding site was defined by the combination of abnormalities on the HRCT scan (ground-glass opacities or alveolar consolidation, cavitations or mass) and localized active bleeding or a clot or bronchial lesion on FOB. The HRCT has revolutionized medicine because it allows doctors to see diseases that, in the past, could often only be found at surgery or at autopsy. HRCT is noninvasive, safe and well-tolerated. It provides a highly detailed look at many different parts of the body. If you are looking at a standard x-ray image or radiograph (such as a chest x-ray), it appears as if you are looking through the body. CT and MRI are similar to each other, but provide a different view of the body than an X-ray does. MRI uses a magnetic field and radio waves to produce images, while CT uses X-rays to produce images. Plain $\mathrm{x}$-rays are an inexpensive, quick exam and are accurate at diagnosing things such as pneumonia, arthritis and fractures. Studies have shown that HRCT appears to be superior to the Chest Radiograph (CXR) for early detection and characteristics of a variety of lung disease. Furthermore, the extent of the disease involvement is shown more accurately on HRCT thus raising the level of confidence for diagnosis of infiltrative lung disease [1].

The HRCT has also been used to guide and to provide information regarding an optimum site for open lung surgery. HRCT provides additional useful information to the CXR and directly influence the clinical management of pediatric patients with pulmonary disease, especially in detecting post-infection or post bone marrow transplant sequelae, bronchiectasis and in evaluating patients with nonspecific CXR findings. Use of the subsecond multislice CT scanner for HRCT of the chest in children provides images of diagnostic quality due to the short imaging time [6], [7].

Ground Glass Nodules (GGNs) are, for example, radiographic appearances of hazy lung opacities not associated with an obscuration of underlying vessels. GGNs come in two forms, "pure" and "mixed". Pure GGNs do not consist of any solid components, whereas mixed GGNs consist of some solid components. GGNs are more clearly shown in High Resolution Computed Tomographic (HRCT) images than plain radiographs. GGNs also appear differently than solid nodules in HRCT images because solid nodules have a higher contrast and well defined boundaries. In addition, the appearance of GGNs in HRCT images is a highly significant finding as they often indicate the presence of an active and potentially treatable process such as bronchioalveolar carcinomas or invasive adenocarcinoma. Because GGNs are typically associated with active lung disease, the presence of GGNs often leads to further diagnostic evaluation, including, for example, lung biopsy. Thus, a computer-based segmentation can be of assistance to medical experts for diagnosis and treatment of certain types of lung disease. Accordingly, there is a need for a system and method of computer-based segmentation that can be used to accurately and consistently segment GGNs for quick diagnosis. Furthermore, the extent of the disease involvement is shown more accurately on HRCT thus raising the level of confidence for diagnosis of infiltrative lung disease. The HRCT has also been used to guide and to provide information regarding an optimum site for open lung surgery. HRCT provides additional useful information to the CXR and directly influence the clinical management of pediatric patients with pulmonary disease, especially in detecting post-infection or post bone marrow transplant sequelae, bronchiectasis and in evaluating patients with nonspecific CXR findings. Use of the sub second multislice CT scanner for HRCT of the chest in children provides images of diagnostic quality due to the short imaging time [11].

Gabor filters: Gabor filters can serve as excellent band-pass filters for one-dimensional signals (e.g., speech). Gabor filters are implemented in image representation, texture segmentation, edge detection, retina identification, image coding and image retrieval in decades. This Gabor filters are preferred for medical image processing and for signal processing applications because of their sharp cutoffs. So, the noise level decreases as the cutoff of the filter smoothes. Gabor filters are also linear shift invariant and orientation selective filters. This can achieve the minimum restriction for simultaneous localization in both spatial and frequency domains. An advantage of these filters is that they satisfy the minimum spacebandwidth product per the uncertainty principle. Hence, they provide simultaneous optimal resolution in both the space and spatial-frequency domains. A Gabor filter is a linear filter used in image processing for edge detection. Its impulse response is defined by a harmonic function multiplied by a Gaussian function. Because of the multiplication-convolution property (Convolution theorem), the Fourier transform of a Gabor filter's impulse response is the convolution of the Fourier transform of the harmonic function and the Fourier transform of the Gaussian function.

This family of filters is used to obtain parts of the image frequency spectrum:

$$
\begin{aligned}
G(x, y) & =\frac{1}{2 \pi \sigma_{x} \times \sigma_{y}} \exp \left[-1 / 2\left(x^{2} / \sigma_{x}^{2}\right)\right. \\
& \left.\left.+\left(y^{2} / \sigma_{y}^{2}\right)+j 2 \pi(x U+y V)\right)\right]
\end{aligned}
$$


Where, $\sigma_{\mathrm{x}}$ and $\sigma_{\mathrm{y}}$ are the standard deviations of the Gaussian functions. $\mathrm{U}$ and $\mathrm{V}$ are the centre frequency of the Gabor filter. The center frequency of each filter was selected to correspond to a peak in the texture power spectrum and filter bandwidths are determined by the center frequency. Gabor filters are directly related to Gabor wavelets, since they can be designed for number of dilations and rotations.

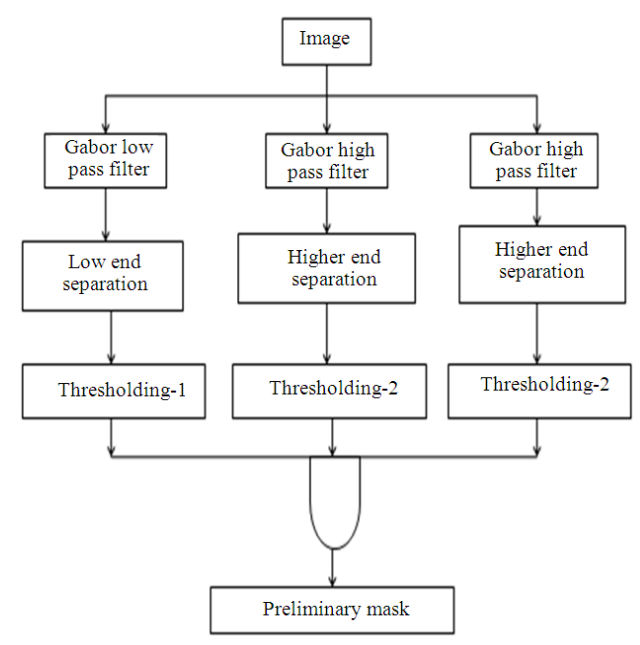

Figure 1. Preliminary mask formation

However, in general, expansion is not applied for Gabor wavelets, since this requires computation of biorthogonal wavelets, which may be very time-consuming. Therefore, usually, a filter bank consisting of Gabor filters with various scales and rotations is created. The filters are convolved with the signal, resulting in a so-called Gabor space. This process is closely related to processes in the primary visual cortex. A Gabor filter is obtained by modulating a sinusoid with a Gaussian. For the case of one Dimensional (1D) signals, a 1D sinusoid is modulated with a Gaussian. This filter will therefore respond to some frequency, but only in a localized part of the signal. Two dimensional Gabor filter is obtained by combining the signal with sinusoid. Two dimensional are widely used in image processing, computer vision, neuroscience and psychophysics. $G$ $(\mathrm{x}, \mathrm{y}, \theta, \varphi)$ be the function defining a Gabor filter centered at the origin with $\theta$ as the spatial frequency and $\varphi$ as the orientation [2]-[4].

Gabor filter bank: Gabor filter bank covers all the wanted frequencies and orientations. The Gabor functions form a complete but non-orthogonal basic set. The term $\mathrm{G}(\mathrm{x}, \mathrm{y})$ is a modulating Gaussian:

$$
\begin{aligned}
& \mathrm{G}(\mathrm{x}, \mathrm{y})=\exp \frac{\left(-\mathrm{x}^{2}+\gamma_{\mathrm{y}}^{2}{ }^{2}\right)}{2 \sigma^{2}} \cos [2 \pi(\mathrm{x} / \lambda)+\phi) \\
& \mathrm{x}^{\prime}=\mathrm{x} \cos \theta+\mathrm{y} \cos \theta \\
& \mathrm{y}^{\prime}=-\mathrm{x} \sin \theta+\mathrm{y} \sin \theta
\end{aligned}
$$

In this above expression, $\sigma$ and $\lambda$ denote variance of the Gaussian function, wavelength of the sinusoidal function respectively. The spatial aspect ratio of the Gabor function is specified by $\gamma$ and by changing this value, the filter output is varied. Accurately low pass and many high pass filter outputs are extracted by changing the value of this spatial aspect ratio. The coordinates are rotated by $\theta$ with respect to the $\mathrm{x}$-axis. The loss of information can be minimized by using the knowledge of the location and orientation of the interface. The aspect ratio is changed by the Gaussian envelope of Gabor filters.

Wavelength $(\lambda)$ : This is the wavelength of the cosine factor of the Gabor filter kernel and herewith the preferred wavelength of this filter. Its value is specified in pixels. Valid values are real numbers equal to or greater than 2 . The value $\lambda=2$ should not be used in combination with phase offset $\varphi=-90$ or $\varphi=90$ because in these cases the Gabor function is sampled in its zero crossings. In order to prevent the occurrence of undesired effects at the image borders, the wavelength value should be smaller than one fifth of the input image size. 
Orientations ( $(\boldsymbol{\theta})$ : The phase offset $\varphi$ in the argument of the cosine factor of the Gabor function is specified in degrees. Valid values are real numbers between 180 and 180 . The values 0 and 180 correspond to center symmetric 'centeron' and 'centeroff' functions, respectively, while 90 and 90 correspond to antisymmetric functions. All other cases correspond to asymmetric functions.

Phase offset(s) $(\varphi)$ : The phase offset $\varphi$ in the argument of the cosine factor of the Gabor function is specified in degrees. Valid values are real numbers between -180 and 180 . The values 0 and 180 correspond to center-symmetric 'center-on' and 'center-off' functions, respectively, while -90 and 90 correspond to antisymmetric functions. All other cases correspond to asymmetric functions.

Aspect ratio $(\gamma)$ : This parameter, called more precisely the spatial aspect ratio, specifies the ellipticity of the support of the Gabor function. For $\gamma=1$, the support is circular. For $\gamma<1$ the support is elongated in orientation of the parallel stripes of the function. Default value is $\gamma=0.5$.

Bandwidth (b): The half response spatial frequency bandwidth $\mathrm{b}$ (in octaves) of a Gabor filter is related to the ratio $\sigma / \lambda$, where $\sigma$ and $\lambda$ are the standard deviation of the Gaussian factor of the Gabor function and the preferred wavelength, respectively.

Ground glass opacity disease: Ground-glass refers to the HRCT appearance of a hazy opacity that does not obscure the associated pulmonary vessels. This appearance results from parenchymal abnormalities that are below the spatial resolution of HRCT. Ground-glass opacity can be seen with alveolar wall inflammation or thickening, with partial air-space filling, or with some combination of the two.

Ground glass opacity is a frequent but nonspecific finding on high-resolution CT scans of the lung parenchyma. The underlying abnormality is diverse; any condition that decreases the air content of the lung parenchyma without totally obliterating the alveoli can produce ground glass opacity. These processes are not visible on high-resolution CT scans. However, in specific clinical settings, the information provided by the high resolution CT is considerable when the anatomic distribution and associated structural changes to the lung parenchyma are analyzed.

The so-called ground glass pulmonary opacity is characterized by a slight increase in lung density, with persistent visibility of vascular structures and bronchial walls. If vessels are obscured, the term consolidation is preferred. This kind of pulmonary opacity, which may be patchy or diffuse, was well known in conventional radiology, but has been recently re-evaluated, following the increasingly widespread use of high resolution CT of the lung. Ground glass opacity is commonly observed in patients with early diffuse pulmonary infiltrative diseases. Though non-specific in itself, the sign is always very significant. Particularly, it could represent a useful sign of active and treatable abnormality in some diffuse pulmonary diseases, such as idiopathic pulmonary fibrosis and sarcoidosis [5].

Ground glass opacity is observed when histological thickening of the alveolar walls and septal interstitium is minimal or the alveolar lumen is partially filled with fluid, macrophages, neutrophils or the amorphous material. The degree of increased lung opacity is not sufficient to obscure pulmonary vessels, as would be the case in true consolidation. Ground-glass opacity is potentially reversible with appropriate therapy, if the underlying disorder is treated early, because none of the changes in lung structure are permanent. Some active but potentially reversible processes that produce ground-glass opacity include pulmonary edema; alveolar proteinosis; and many causes of alveolitis or interstitial fibrosis, sarcoidosis, hypersensitivity pneumonitis and early radiation pneumonitis.

Ground glass opacity may also be seen as a consequence of increased capillary blood volume in redistribution of blood flow. Although it is a non specific finding, it can suggests a specific diagnosis in certain clinical circumstances or indicate a potentially treatable disease. Beyond that it is an initial appearance of the diffuse lung disease. Therefore, accurate detection and differential diagnosis of ground glass opacity are important. The currently available lung disease detection algorithms are based on statistical learning [8]-[10].

Significance of ground glass opacity: In some diseases, such as idiopathic pulmonary fibrosis (fibrosing alveoliis) and sarcoidosis, the appearance of ground glass opacity correlates with sdisease activity, as indicated by biopsy, bronchioalveolar lavage, or gallium lung scans. These areas of ground-glass opacity have been shown to correspond to regions of active alveolitis and precede irreversible changes such as fibrosis and honey-combing. It has been reported that ground-glass opacity can be receiving steroids. Some authors believe that the presence of ground-glass opacities correlates with disease activity and thus indicates which patients should receive steroids. High resolution CT, therefore, should also be used to guide biopsies to areas of ground-glass opacity rather than to fibrotic areas of inactive disease.

Anatomic distribution of ground glass opacity: A centilobular distribution indicates early airspace consolidation, which may be due to bronchial dissemination of either infection or blood. Hypersensitivity pneumonid desquarmative interstitial pneumonitis also can manifest as centilobular ground-glass opacity. The term air-space nodule is used in conventional radiography and high resolution CT to describe poorly defined nodular opacities ranging in diameter from a few millimeters to $1 \mathrm{~cm}$. Airspace nodules represent peribronchiolar areas of air-space consolidation. 
A pan lobular distribution of ground-glass opacity can sharply demarcate a diseased secondary pulmonary lobule from unaffected, normally aerated neighboring parenchyma. The boundaries between normal and abnormal areas will be indistinct if only portions of a secondary pulmonary lobule are diseases. When ground-glass opacity is found to involve a large area, either in a patchy or homogenous, lobar pattern, many diagnoses can be excluded, because those diseases usually progress to complete consolidation. Possible diagnoses include alveolar proteinosis, dug toxicity, liquid pneumonia, sarcoidosis and pneumocystis carinii pneumonia. Resolving pneumonia or hemorrhage also many manifest as ground-glass opacity.

\section{Ground glass detection masking:}

Preliminary mask formation: In this preliminary mask formation, the filters output are extracted from the Gabor filter bank. Low pass filter is only allowed the local average intensity information. By the following form, the lower frequency spectrum is extracted from Gabor filters.

$$
\mathrm{G}(\mathrm{x}, \mathrm{y})=\frac{\mathrm{x}+\mathrm{y}}{2 \pi \sigma} \exp \left[-1 / 2\left(\left(\mathrm{x}^{2}+\mathrm{y}^{2}\right) / \sigma^{2}\right]\right.
$$

In low pass filtering all regions are unable to be differentiated but the high pass filters successfully filter out increased opacity patterns. Because Gabor filters are band pass filters it is used to separate the images of low and high frequencies. They also pick up large nodules. This appears as large bright blobs which are similar to rectangular pulses. They cannot be filtered out because it has large sinc frequency spectrum. Although in the low pass filter output nodules appear and it can be easily discarded by thresholding.

The process of thresholding is applied to the images of different frequencies and the and operation is performed on the images to obtain the preliminary mask, but still the images are not clear, it is accompanied by some noise. So binary noise removal technique is used in the next section to remove noise.

\section{Steps involved:}

Thresholding: Partitioning images directly into images based on intensity values and /or properties of these values is called thresholding. During the thresholding process, individual pixels in an image are marked as "object" pixels if their value is greater than some threshold value (assuming an object to be brighter than the background) and as "background" pixels otherwise. This convention is known as threshold above. Variants include threshold below, which is opposite of threshold above; threshold inside, where a pixel is labeled "object" if its value is between two thresholds; and threshold outside, which is the opposite of threshold inside Typically, an object pixel is given a value of " 1 " while a background pixel is given a value of "0." Finally, a binary image is created by coloring each pixel white or black, depending on a pixel's label.

Adaptive thresholding: Thresholding is called adaptive thresholding when a different threshold is used for different regions in the image. This may also be known as local or dynamic thresholding. The key parameter in the thresholding process is the choice of the threshold value. Several different methods for choosing a threshold exist; users can manually choose a threshold value, or a thresholding algorithm can compute a value automatically, which is known as automatic thresholding. A simple method would be to choose the mean or median value, the rationale being that if the object pixels are brighter than the background, they should also be brighter than the average. In a noiseless image with uniform background and object values, the mean or median will work well as the threshold, however, this will generally not be the case. A more sophisticated approach might be to create a histogram of the image pixel intensities and use the valley point as the threshold. The histogram approach assumes that there is some average value for the background and object pixels, but that the actual pixel values have some variation around these average values. However, this may be computationally expensive and image histograms may not have clearly defined valley points, often making the selection of an accurate threshold difficult. One method that is relatively simple, does not require much specific knowledge of the image and is robust against image noise, is the following iterative method.

An initial Threshold (T) is chosen; this can be done randomly or according to any other method desired. The image is segmented into object and background pixels as described above, creating two sets:

$\mathrm{G}_{1}=\{\mathrm{f}(\mathrm{m}, \mathrm{n}): \mathrm{f}(\mathrm{m}, \mathrm{n})>\mathrm{T}\}$ (object pixels)

$\mathrm{G}_{2}=\{\mathrm{f}(\mathrm{m}, \mathrm{n}): \mathrm{f}(\mathrm{m}, \mathrm{n}) \mathrm{T}\}$ (background pixels)

(note, $\mathrm{f}(\mathrm{m}, \mathrm{n})$ is the value of the pixel located in the $\mathrm{m}^{\text {th }}$ column, $\mathrm{n}^{\text {th }}$ row)

The average of each set is computed:

$\mathrm{m}_{1}=$ average value of $\mathrm{G}_{1}$

$\mathrm{m}_{2}=$ average value of $\mathrm{G}_{2}$

A new threshold is created that is the average of $\mathrm{m}_{1}$ and $\mathrm{m}_{2}$ : 
$\mathrm{T}^{\prime}=\left(\mathrm{m}_{1}+\mathrm{m}_{2}\right) / 2$

Go back to step two, now using the new threshold computed in step four, keep repeating until the new threshold matches the one before it (i.e., until convergence has been reached).

This iterative algorithm is a special one-dimensional case of the k-means clustering algorithm, which has been proven to converge at a local minimum-meaning that a different initial threshold may give a different final result.

Categorizing thresholding methods: Thresholding methods can be categorized into the following six groups based on the information the algorithm manipulates.

Histogram shape-based methods, where, for example, the peaks, valleys and curvatures of the smoothed histogram are analyzed Clustering-based methods, where the gray-level samples are clustered in two parts as background and foreground (object), or alternately are modeled as a mixture of two Gaussians Entropy based methods result in algorithms that use the entropy of the foreground and background regions, the crossentropy between the original and binarized image. Object attribute-based methods search a measure of similarity between the gray-level and the binarized images, such as fuzzy shape similarity, edge coincidence.

[...] spatial methods [that] use higher-order probability distribution and/or correlation between pixels. Local methods adapt the threshold value on each pixel to the local image characteristics.

Multiband thresholding: Color images can also be threshold. One approach is to designate a separate threshold for each of the RGB components of the image and then combine them with an AND operation. This reflects the way the camera works and how the data is stored in the computer, but it does not correspond to the way that people recognize color. Therefore, the HSL and HSV color models are more often used. An interesting new method uses the CMYK color model to threshold color images.

In histology images the color is given by 2 or more dyes, for example hematoxylin and DAB .The contributions of these two dyes to the color image can be established by using color deconvolution. The incident light is measured by looking at an unstained part of the slide. Then you measure the relative absorption of each the 3 RGB components of the image by each dye. Absorption is defined by Beer's Law. The absorption characteristics for each dye can be obtained by measuring the RGB components of the color in areas of the slide that are only colored by one dye. The computer software can solve 3 linear equations for each pixel in the image, one for each color channel, in order to resolve the amounts of each of the dyes at each locus in the image. This method can resolve up to 3 different dyes. The result is an image for each dye, showing the amount of that dye throughout the image. This allows the amount of dye to be quantified, which is important for image analysis of immunohistochemical stains.

Edge detection: Edge detection is a fundamental tool used in most image processing applications to obtain information from the frames as a precursor step to feature extraction and object segmentation. This process detects outlines of an object and boundaries between objects and the background in the image. An edge-detection filter can also be used to improve the appearance of blurred or anti-aliased video streams.

Traditionally, the methods used for texture edge detection were based on statistical approaches. There are many disadvantages in using statistical approaches for texture edge detection. The problem with statistical approach is that they do not consider multi-channel, multi resolution approach of human vision system. Gabor filter and Discrete Wavelet Transform (DWT) are two band pass filters that resemble the human visual system. Gabor filters are specially tuned to segment images with bipartite textures.

Edge detection is one of the important preprocessing steps in many of the image processing applications. Various edge detection techniques are available for images on rectangular grids such as classical operators, CLAP algorithm based edge detection, wavelet based edge detection. Wavelet based edge detection is found to be a better technique for specific application such as iris recognition system; 3D vertebrae shape recognition, infrared target recognition. These techniques are not suitable for hexagonal domain. The edge detection is realized by the convolution with a set of directional derivative masks.

The basic edge-detection operator is a matrix area gradient operation that determines the level of variance between different pixels. The edge-detection operator is calculated by forming a matrix centered on a pixel chosen as the center of the matrix area. If the value of this matrix area is above a given threshold, then the middle pixel is classified as an edge. Examples of gradient-based edge detectors are Roberts, Prewitt and Sobel operators. All the gradient-based algorithms have kernel operators that calculate the strength of the slope in directions which are orthogonal to each other, commonly vertical and horizontal. Later, the contributions of the different components of the slopes are combined to give the total value of the edge strength.

Depending on the noise characteristics of the image or streaming video, edge detection results can vary. Gradient-based algorithms such as the Prewitt filter have a major drawback of being very sensitive to noise. The size of the kernel filter and coefficients are fixed and cannot be adapted to a given image. An adaptive edge-detection algorithm is necessary to provide a robust solution that is adaptable to the varying noise levels of these images to help distinguish valid image content from visual artifacts introduced by noise. 
Calculating accuracy:

Sensitivity: Sensitivity of the algorithm is measured by considering each individual diseased region labeled by the radiologists as ground glass and counting the result of Ground Glass detection Algorithm $($ GGDA) Sensitivity = FP / $(\mathrm{FP}+\mathrm{LN})$ :

1. Final Positive (FP): Regions labeled by the radiologist and recognized by GGDA

2. Last positive (LP): Regions recognized by GGDA but not labeled by radiologist

3. Last Negative (LN): Regions labeled by radiologist but not recognized by GGDA

4. Final Negative (FN): Regions not labeled or recognized by either the radiologist or GGDA

Specificity: The specificity of the algorithm, which is one minus the probability of a slice being labeled as ground glass when there is no ground glass present, can be calculated as follows and values are shown in table.

\section{MATERIALS AND METHODS}

In this study Ground glass pattern detection of HRCT images is done for better visualization and their analysis. For this we have taken the HRCT images of lungs from Ground glass opacity patients. These are basically RGB images and these are converted to GRAY SCALE images for making further process easy. Then preliminary mask is obtained using Gabor high pass and Gabor low pass filters. Thresholding and Morphological operations such as erosion and dilation are performed to obtain peripheral mask. This peripheral mask contains noise which appears as tiny dots and they are often few pixels wide. For getting accuracy, we are going for post processing technique in which the noise is removed by median filters. This may assist doctors for making decisions for better and quick treatment. The results obtained from the above steps are shown in the following Figure 2-9.
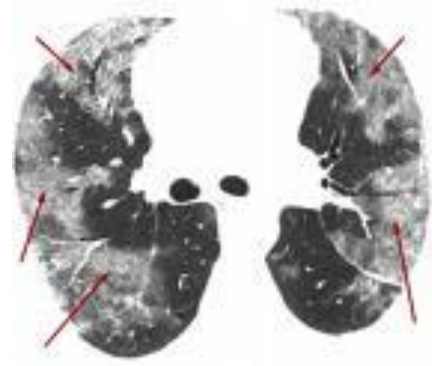

Figure 2. Original image

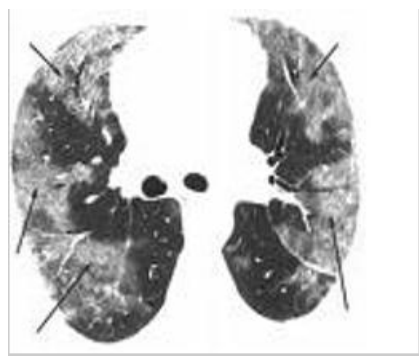

Figure 4. Input Image

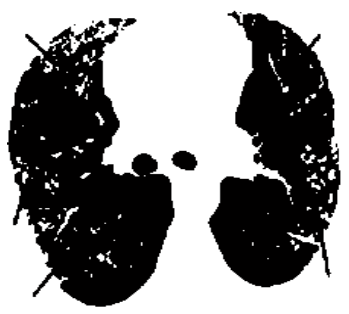

Figure 6. Preliminary mask output

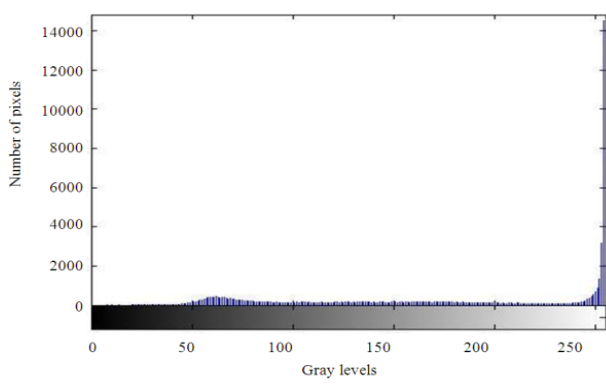

Figure 3. Histogram of original image

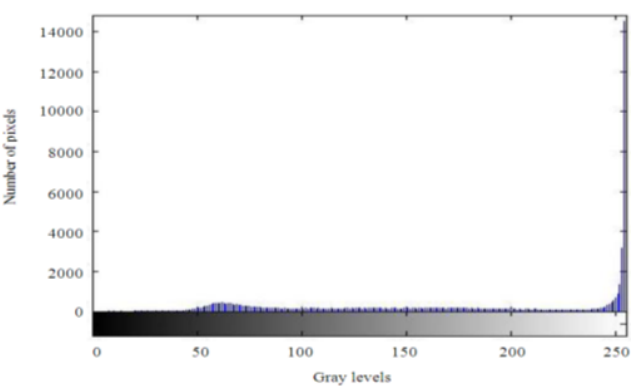

Figure 5. Histogram of Input image

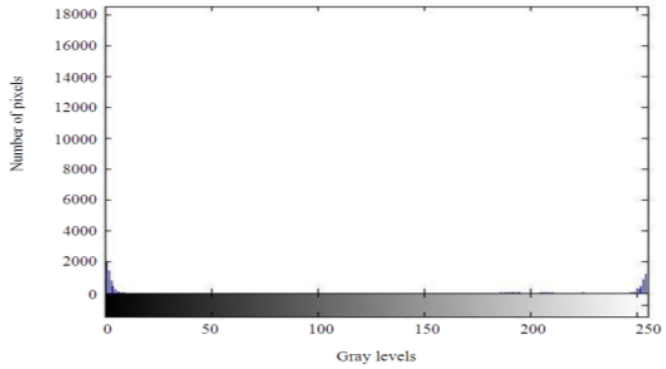

Figure 7. Histogram of preliminary mask

Performance and Analysis of Automatic Detection of Ground-Glass Pattern in Lung... (M. Anto Bennet) 


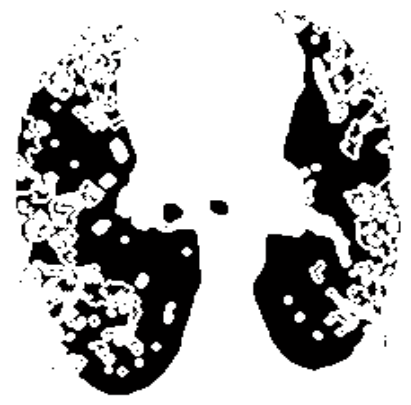

Figure 8. Final Post Processing Output

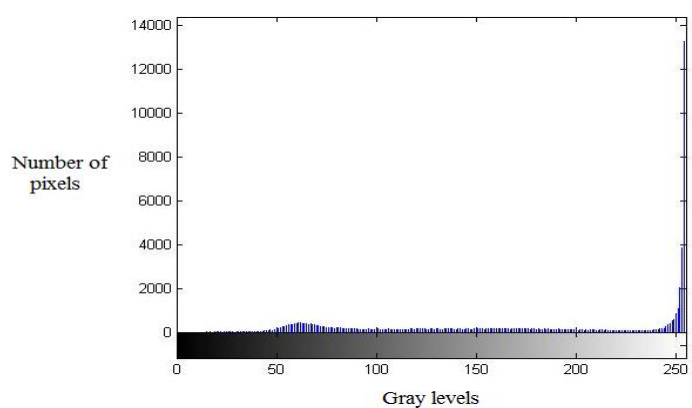

Figure 9. Histogram of Post processing

\section{CONCLUSION}

By the Ground glass detection algorithm, the Gabor filter banks are designed and the masks are formed. Thus, the ground glass pattern is detected using frequency spectrum analysis with minimal post processing. This technique may demonstrate high sensitivity and specificity with fast processing time.

Future scope: Potential future work includes removing noise by various filter techniques. This algorithm may be extended to the detection of other diffuse lung diseases like reticular, consolidation, emphysema; Nodular opacity and honey combing pattern and the sensitivity can be increased by using various thresholding techniques and noise removal techniques.

\section{REFERENCES}

[1] Rahmat M. F., M. D. Isa, K. Jusoff, T. A. Hussin and S. Md. Rozali, "Image Reconstruction Algorithm for Electrical Charge Tomography System", Am. J. Applied Sci., Vol. 7, pp. 1254-1263, 2010.

[2] Noor N. R. M., N. A. M. Isa, M. Y. Mashor, N. H. Othman and K. Z. Zamli, et al., "Automatic Glass-Slide Capturing System for Cervical Cancer Pre-Screening Program”, Am. J. Applied Sci., Vol. 5, pp. 461-467, 2008.

[3] Benamrane N., A. Freville and R. Nekkache, "A hybrid fuzzy neural networks for the detection of tumors in medical images", Am. J. Applied Sci., Vol. 2, pp. 892-896, 2005.

[4] Rizon M., M. F. Hashim, P. Saad and S. Yaacob, "Face recognition using eigenfaces and neural networks", Am. J. Applied Sci., Vol. 3, pp. 1872-1875, 2006.

[5] Murad S. A. Z., and W. Ismail, "Design of active integrated antenna for dual frequency image rejection", Am. J. Applied Sci., Vol. 3, pp. 1890-1894, 2006.

[6] Desai S. R., "High resolution computed tomography of the lung", CME Radiology, Vol. 2, pp. 126-133, 2001.

[7] Fukumoto, H. A. B., T. Nitta and N. T. Sudagawa, "Detection of Ground Glass Opacities in lung CT images using Gabor filters and neural networks", IEEE Trans., Vol. 1, pp. 251-256, 2005.

[8] Hoffman E.A., and A. V. Clough, "The comprehensive imaging based analysis of the lung", Am. Radiol., Vol. 11, pp. 1370-1380, 2004.

[9] Manjunath B. S., and W.Y. Ma, "Texture features for browsing and retrieval of image data", IEEE Trans., Vol. 18, pp. 837- 842, 2006.

[10] Uppaluri R., M. Sonka, P. G. Hartley, G. W. Hunninghake and G. M. Lennan, "Computer recognition of regional lung disease patterns", Am. J. Respiratory Critical Care Med., Vol. 160, pp. 648-654, 1999.

[11] Yamaguchi T., K. Ashizawa, K. Nagaoki and K. Hayashi, "High resolution CT evaluation of ground glass opacity in diffuse lung disease", Acta Med. Nagasaki, Vol. 43, pp. 1-11, 2000. 